NMR in Biomedicine

\title{
Validation of a semi-automatic coregistration of MRI scans in brain tumor patients during treatment follow-up
}

\begin{tabular}{|r|l|}
\hline Journal: & NMR in Biomedicine \\
\hline Manuscript ID & NBM-15-0311.R2 \\
\hline Wiley - Manuscript type: & Research Article \\
\hline Complete List of Authors: & $\begin{array}{l}\text { van der Hoorn, Anouk; University Medical Centre Groningen, Department } \\
\text { of radiology (EB44); University of Cambridge, Department of Radiology; } \\
\text { University of Cmabridge, Brain tumor imaging laboratory } \\
\text { Yan, Jiun-Lin; University of Cambridge, Clinical Neuroscience; Chang Gung } \\
\text { Memorial Hospital, Department of neurosurgery; Chang Gung University } \\
\text { College of Medicine, Department of neurosurgery } \\
\text { Larkin, Timothy; University of Cambridge, Brain tumor imaging laboratory } \\
\text { Boonzaier, Natalie; University of Cambridge, Brain tumor imaging } \\
\text { laboratoy } \\
\text { Matys, Tomasz; University of Cambridge, Department of Radiology } \\
\text { Price, Stephen ; University of Cambridge, Brain tumor imaging laboratory }\end{array}$ \\
\hline Keywords: & $\begin{array}{l}\text { brain tumors, high grade gliomas, MRI, treatment response, validation, } \\
\text { coregistration }\end{array}$ \\
\hline
\end{tabular}


Anouk van der Hoorn, MD PhD ${ }^{1-3+}$; Jiun-Lin Yan, $\mathrm{MD}^{1,4-5 \dagger^{*}}$; Timothy $\mathrm{J}$ Larkin, $\mathrm{PhD}^{1}$;

Natalie R Boonzaier, $\mathrm{MSc}^{1}$, Tomasz Matys, MD PhD²; Stephen J Price, BSc

MBBS(Hons) PhD FRCS(Neuro Surg. $)^{1}$

${ }^{\dagger}$ contributed equally;

${ }^{1}$ Brain tumor imaging lab, Division of neurosurgery, Department of clinical neuroscience, University of Cambridge, Addenbrooke's hospital, Box 167, CB2 0QQ, Cambridge, United Kingdom;

${ }^{2}$ Department of radiology, University of Cambridge, Addenbrooke's hospital, Box 218, CB2 OQQ, Cambridge, United Kingdom;

${ }^{3}$ Department of radiology (EB44), University Medical Centre Groningen, University of Groningen, Box 30.001, 9700 RB, Groningen, The Netherlands;

${ }^{4}$ Department of neurosurgery, Chang Gung Memorial Hospital, 22 Majing road, 204, Keelung, Taiwan

${ }^{5}$ Department of neurosurgery, Chang Gung University College of Medicine, 259

Wenhua $1^{\text {st }}$ road, 333, Taoyuan, Taiwan

Corresponding author:

Jiun-Lin Yan

Division of neurosurgery

Department of Clinical neuroscience

University of Cambridge

Addenbrooke's hospital

Box 167

CB2 0QQ

Cambridge, United Kingdom

Running title: Coregistration of MRI in brain tumor patients

Word count: 4342

Figures: 5

Tables: 2

Supporting material for online-only publication: Yes 


\begin{abstract}
There is an expanding research interest in high grade gliomas due to their significant population burden and poor survival despite the extensive standard multimodal treatment. One of the obstacles is the lack of individualized monitoring of tumor characteristics and treatment response before, during and after treatment. We developed a two stage semi-automatic method to coregister MRI scans at different time points before and after surgical and adjuvant treatment of high grade gliomas. This two stage coregistration includes a linear coregistration of the semi-automatically derived mask of the preoperative contrast enhancing area or postoperative resection cavity, brain contour, and ventricles between different time points. The resulting transformation matrix was then applied to a nonlinear manner to coregister conventional contrast-enhanced T1-weighted images. Targeted registration errors were calculated and compared to linear and nonlinear coregistered images. Targeted registration errors were smaller for the semi-automatic non-linear coregistration compared with both the nonlinear and linear coregistered images. This was further visualized utilizing a 3D structural similarity method. The semi-automatic non-linear coregistration allowed for optimal correction of variable brain shift at different time points as evaluated by minimal targeted registration error. This proposed method allows for accurate evaluation of treatment response, essential for the growing research area of brain tumor imaging and treatment response evaluation in large sets of patients.
\end{abstract}

Keywords: Linear coregistration; nonlinear coregistration; brain tumors; high grade gliomas; MRI; treatment response; validation; structure similarity; 


\section{INTRODUCTION}

Despite advancements in treatment of high grade gliomas, including the introduction of concomitant chemoradiotherapy and adjuvant chemotherapy (1), these tumors continue to carry a high mortality rate and significant population burden (2-3). One of the unmet needs in treatment development research is the ability to easily identify differences in tumor characteristics and treatment response with MRI imaging biomarkers before, during and after therapy. This would facilitate research on treatment response in large sets of patients and discovery of new imaging biomarkers, further enabling personalization of therapy. In order to meet this demand, an easily applicable coregistration method is needed to coregister postoperative with preoperative images during MRI assessment of high grade gliomas.

A nonlinear registration is needed as brain shift and deformation pose significant challenges when comparing brain MR images at different time points. These changes do note only occur after the initial surgical procedure, but are rather a dynamic continuous process (4). In addition to the structural changes caused by surgery, tumor response to treatment and chronic radiotherapy effects induce further changes. These include, for example, changes in tumor volume (5), making the coregistration process even more difficult.

Though previous research has aimed to address these obstacles, an easily accessible and applicable coregistration method is not yet available for preoperative and postoperative MRI scans of patients with brain tumors. Most methods focus on coregistration of different sequences of the same scan time point with linear and nonlinear registration methods in healthy subjects and brain tumor patients. These existing methods demonstrate good performances for this intrasubject coregistration 


\begin{abstract}
of data from the same time (6-7) or to a standardized brain atlas (8-9). Research has also demonstrated the value of a nonlinear coregistration for treatment response evaluation in patients without surgery (10). However, surgery is part of the standard treatment scheme in patients with high grade gliomas (1) and is regularly performed in other primary and metastatic brain tumors (11-12). Therefore, the ability to deal with post-surgical changes should be part of the coregistration method.
\end{abstract}

Methods of MRI coregistration taking resection into account are scarce and have several limitations making them unsuitable as a widely and easily usable coregistration method. The few available clinical studies that have used an intrasubject coregistration method after surgery are difficult to replicate and evaluate as they use in-house software, and provide only limited details of methodology (e.g. 13-15). Technical studies suffer from other issues, such as small sample size (16). Another study has tested the methods only on epilepsy patients with whole lobe resection (17), where less signal changes and mass effects are expected. Furthermore, these and other technical papers do not provide comprehensible coregistration guideline and thereby hinder wide applicability, especially for clinical researchers (16-18).

There are a few methods available that coregister intraoperative images with preoperative imaging. However, these are limited by using other modalities, like CT (19), tracked laser range scanning (20) or ultrasound (21). CT is inferior to MRI in detecting tumor recurrence and thus not routinely used for the treatment evaluation or in research of brain tumor patients. Ultrasound and laser imaging are only possible during surgery when the skull is temporarily removed. The method from Nithiananthan and colleagues (19) uses an approach that defines resected voxels 
based on an air density. This is not applicable to research with interest in tumor response assessment using postoperative $\mathrm{MRI}$ imaging as the resection cavity being filled with fluid and/or adjacent brain tissue. Other preoperative with intraoperative coregistration methods have only tested the complex algorithm on 2D data (22).

Therefore, a need for an easily applicable and usable coregistration method before, during and after treatment including surgical resection is clearly required. To address this, we developed a semi-automatic coregistration technique using widely used and freely available software. This may allow accurate evaluation of treatment response in future studies, which is essential for brain tumor imaging research and treatment response assessment in large sets of patients. We also provide detailed information about the steps and the code used. 


\section{METHODS}

\section{Patient inclusion criteria}

Patients with newly diagnosed supratentorial glioblastoma, operated from 2010 to 2014, were included in the study. Exclusion criteria were previous cranial surgery, previous cerebral radiotherapy or another known primary tumor. We included 32 patients (mean age 56 years, range 31-68; 20 males) with preoperative MRI scans that also had available follow-up MRI. Follow-up MRI scans included a direct postoperative scan and the scan at the time-point of tumor recurrence. Adequate direct postoperative MRI data ( $<72$ hours) was available for 30 patients, while later follow-up at the time of tumor recurrence was available for 27 patients (mean 12 months after the operation date, range 0.8-38). All patients were treated with maximal surgical resection followed by standard concomitant chemoradiotherapy followed by adjuvant chemotherapy (1).

The study was approved by the local Institutional Review Board (10/H0308/23) and informed written consent was obtained from all patients.

\section{Data acquisition}

Preoperative MRI data acquisition was performed using a 3.0 T Siemens Magnetom MRI system (Siemens Healthcare, Munich, Germany) with a standard 12-channel head coil. Imaging included an anatomical 3D T1-weighted sequence with fat suppression acquired after the intravenous injection of $9 \mathrm{~mL}$ gadolinium (Gadovist, Bayer Schering Pharma, Berlin, Germany) (TR/TE 900/2.98 ms, inversion time 900 ms; flip angle $9^{\circ}$, FOV $256 \times 240$ mm; 176-208 slices; no slice gap; voxel size $1 \times 1 \times$ $1 \mathrm{~mm})$. 
Follow-up MRI scans were acquired on a 1.5 T GE Optima, 1.5 or 3.0 T GE Signa (General Electric Company, Little Chalfont, United Kingdom) or 1.5 T Siemens Avanto (Siemens Healthcare, Munich, Germany) with standard head coil. Imaging included a T1-weighted anatomical sequence after the intravenous injection of $9 \mathrm{~mL}$ gadolinium (Gadovist, Bayer Schering Pharma, Berlin, Germany). This was performed as a 2D T1-weighted sequence (TR/TE 440-771/8-21 ms, flip angle 58-90, FOV 220-240 x 207-240 mm; 20-85 slices; 0-1 mm slice gap; voxel size $0.429-0.7188 \times 0.429-0.7188 \times 3-6 \mathrm{~mm})$, a $2 \mathrm{D} \mathrm{T} 1$ inversion recovery sequence (TR/TE 2508-2600/12-42 ms, inversion time 780-920 ms; flip angle 90-110 ${ }^{\circ}$ FOV $220 \times 220 \mathrm{~mm} ; 20-22$ slices; $1-3.5 \mathrm{~mm}$ slice gap; voxel size of $0.4297 \times 0.4297 \times 6$ $\mathrm{mm}$ ) or a 3D T1 fat suppressed sequence (TR/TE 7/2.948 ms, inversion time $900 \mathrm{~ms}$; flip angle $190^{\circ}$, FOV $256 \times 256$ mm; 190 slices; no slice gap; voxel size 1 x 1 x $1 \mathrm{~mm}$ ).

\section{Coregistration method}

Coregistration was performed using a two-staged semi-automatic method (Figure 1). Conventional contrast-enhanced T1-weighted images were coregistered using tools from the FMRIB Software Library (FSL) version 5.0 .0 (http://fsl.fmrib.ox.ac.uk/fsl/fslwiki/). Before the coregistration stages commence, all images were realigned with the anterior commissure as the center point (coordinate 0 , $0,0)$ to minimize the influence of brain position. The first stage was the coregistration of the binary masks, which consisted of the outer contour of the brain, the ventricles, and contrast enhancing area (presurgical MRI images) or resection area (follow up MRI images). This was performed for each subject at the different time points to create a transformation matrix (Figure 1, step 4, using the FLIRT, FMRIB's linear image registration tool, function). The brain contours were created from the inversion of the brain masks which were semi-automatically extracted (23). This was followed by 
The second stage applied the transformation matrix, acrequired from the first stage, as input for a nonlinear transformation matrix of the brain images (Figure 1, step 5 and 6, using the FNIRT, FMRIB's non-linear image registration tool, functions). The nonlinear transformation of the brain images used additional subsampling levels for regularization. This also included the binary masks of the brain contour and ventricles from both the preoperative reference image and the follow-up image. This resulted in a coregistration of the follow-up brain extracted MRI scans with the preoperative brain extracted MRI scans. The steps with accompanying FSL code are illustrated (Figure 1) and are provided as supporting material to facilitate use by others (see supporting material). Standard linear coregistration (FLIRT function) and standard FNIRT with default setting were done separately for comparison using the FLIRT and FNIRT option in FSL.

\section{Validation methods}

Validation was performed using a targeted registration error method for calculating the error in different directions. Validation in the $\mathrm{x}$ and $\mathrm{y}$ directions was performed utilizing the cerebral aqueduct and septum pellucidum on the same axial slice (Figure 
2A). Validating in the $y$ and $z$ directions (Figure 2B) was performed utilizing the upper anterior boundary of the third ventricle, at the level of the foramen of Monro (Figure $2 \mathrm{C}$ ), on the same coronal slice. The central point of the tumor or cavity was targeted automatically for the calculation of the registration error at the location where most errors could be expected. Vectors were also calculated for all targets. All targeted registration errors were calculated for the semi-automatic non-linear coregistration method and compared to the targeted registration errors for the linear coregistered and standard non-linear coregistered images. Differences were tested with a Wilcoxon signed rank test or paired t-test depending on the normality. Two-sided $p$-values were used.

In addition, a 3D structural similarity map was created (25) using Matlab (MathWorks Inc., Natick, MA, USA). The 3D structural similarity map was created for each subject comparing the preoperative reference scan with the coregistered follow-up scan. This was done for scans obtained direct postoperative and at tumor recurrence separately. To display group results, the preoperative images were coregistered to standard MNI (Montreal Neurological Institute) space with a nonlinear transformation of the brain images including a lesion mask. This was preceded by a linear transformation of a binary mask of the brain exterior and ventricles. A mean structural similarity mask was then created by transforming the structural similarity mask of each patient to standard MNI space. The resection cavity or contrast enhancing areas were excluded for the mean structural similarity image, as these values are inherently different. One author (JLY) also visually inspected all the coregistered MRI scans for accuracy. 


\section{RESULTS \\ Targeted registration error}

The targeted registration error showed good performance of the coregistration method for the direct postoperative and recurrence images with a clear benefit over the linear coregistration method and standard FNIRT non-linear coregistration method (Table 1). In the coregistration of postoperative to preoperative images, $\underline{\text { in }}$ comparison to the FLIRT, the SAC non-linear coregistration showed a smaller deviation of vector deviation of the cerebral aqueduct (1.1 versus $1.6, p=0.015)$. A smaller deviation was also detected for the-, septum pallucidum y coordinate and vector ( 1.3 versus $2.0, p=0.029 ; 1.8$ versus $2.6, p=0.021)$, as well as theand upper most of third ventricle $\mathrm{y}, \mathrm{z}$ coordinate and vector $(0.4$ versus $2.2, p<0.001 ; 1.2$ versus $1.9, p=0.043 ; 1.3$ versus $3.3, p<0.001)$. The semi-automatic nonlinear coregistration also outperformed the default FNIRT coregistration, where, there was small deviation between preoperative and postoperative images small deviation can be seen in for most of the coordinates and vectors in the central tumor/cavity point, cerebral aqueduct, septum pellucidum and the third ventricle at the level of foramen of Monro.

The benefit of this coregistration method can also be seen in the coregistration of recurrent images to preoperative images (Table 2). The target error at The mean error for__the cerebral aqueduct_and-and thethe- roof of the third ventricle atat the level of foramen of Monro was smaller-both displayed smaller orrors for using the semi-automatic non-linear coregistration than the linear coregistration and FNIRT coregistration. When using default FNIRT, there was a larger $z$ coordinate deviation at the tumor centroid point. Additionally, the linear coregistration failed in three patients, and default FNIRT coregistration failed in 2 patients, for both the 
postoperative and recurrence scan, while the semi-automatic method was used without problems.

\section{D structural similarity}

Mean 3D structural similarity of all 32 patients showed the relative performance of the coregistration method (Figure 3), which is also illustrated using a representative subject using contour overlay images (Figure 4). The peripheral areas, including the frontal and parietal areas, demonstrated the best performance. A good performance was also seen at the periventricular regions. A relatively lower overlap between the coregistered and reference preoperative scan was seen in the midsagittal and central areas, the centrum semiovale and central cerebellum.

An example of this coregistration iswas shown in figure 5. Without proper coregistration, one cannot confidently compare the initial postoperative andor tumour recurrencet images ean hardly be compared with-with the pre-operative reference images (Figure 5A). FAlthough - using the standard FNIRT coregistration offor postoperative MR and recurrent MR coregistration (Figure 5B) realigned images resulted in the same space as the reference images-realignment, regional torsions (white arrows) were detected at the resection edges. By using the semi-automatic coregistration methods, the coregistered postoperative MR (Figure $5 \mathrm{C}$ left) and the recurrent MR (Figure $5 \mathrm{C}$ middle) were able to show the residual contrast enhanced lesion (yellow contour) and the recurrent area (red contour) in the reference $\mathrm{MR}_{\text {. }}$ in this case-(Figure 5C). 


\section{DISCUSSION}

We developed and validated a two staged semi-automatic method for the coregistration of preoperative and follow-up MRI scans for both the direct postoperative and tumor recurrence time point (Table1, Table 2, and Figure 5). The semi-automatic derived mask of the outer brain contour, ventricles and lesion allowed an accurate coregistration despite the changes in brain shift and postoperative changes. Therefore, this method is highly applicable to analyze large imaging datasets to evaluate treatment response, a growing and clinically important research area. Furthermore, it can be easily reproduced, allowing a wide applicability of the method.

In the standard FNIRT function, an affine transformation is required as a starting guess of the coregistration. This affine transformation is typically the result of linear FLIRT coregistration between different MR series. However, the deformation in different time points happens mainly over the peritumoral regions, resection cavities, and ventricles. Our approach was to use the transformation affine of these areas with the greatest changes rather than the whole brain images (Figure 1 step 4). Furthermore, we added an inverted brain mask as an outer frame to our first stage coregistration for the gross spatial position (Figure 1 step 2 and 3 ). This resulting affine was further applied to the normal brain parenchyma with the mask of ventricle and lesion (Figure1 step 5) to achieve the optimal coregistration.

To the best of our knowledge, there is a shortage of easily applicable methods that allow coregistration of preoperative and postoperative imaging in patients with brain tumors. Clinical papers often use in-house software solutions and provide only partial description of the methodology (e.g. 13-15). This hinders reproducibility and makes 
independent evaluation of reliability difficult. On the other hand, technical papers are often complex and use only a small number of patient datasets, up to two patients with brain tumors (16) and up to six patients with epilepsy surgery (17). We created an easily reproducible method (see online-only material for the code) and, tested it in a large $(N=32)$ population. Previous studies utilizing intraoperative coregistration have shown a clear advantage of nonlinear coregistration with high diagnostic and stereotactic accuracy $(10,26)$. This supports the results of the postoperative coregistrations from our study.

This two-staged semi-automatic coregistration method can be replicated by using the FMRIB Software Library which is a freely available and widely used software package in the neuroimaging research community. We have provided a detailed description of the steps required to recapitulate this approach (see Figure 1 and supporting material). This makes this method easily reproducible by others, including clinical researchers, which further supported the rationale of using FSL for our coregistration method. Given this purpose of this study, we have not tried to validate the coregistration of patients with brain tumors using other software packages. Interpolation of our semi-automatic method to other software packages might be possible, but would require separate validation.

The MR image acquisition parameters in our dataset varied across subjects and time points. In particular, a contrast-enhanced 3D T1-weighted dataset was available for the preoperative scan, but the follow-up images were almost always 2D and obtained with different MRI parameters and scanners from different manufacturers. Another limitation is that this method assumes that the resection cavity is a result of surgical resection of contrast enhancing tumour only, and that the recurrent tumour arises 


\section{from non-contrast enhancing surrounding area. resulted mostly from the gross tumor} lesion and that the recurrent tumor arises from the peritumoral area. However, the resection volume may goextend beyond the area of contrast enhancement to include the 'peri-tumoural region' $d$ area $_{2}$ particularlyespecially if the resection is conducted under the fluorescence guidance of 5 aminoleuvulinic acid (27). Despite these differences, our validation showed that the method worked in all subjects, making it easily applicable in clinical practice.

At a time when adequate imaging biomarkers are being sought to evaluate treatment response, method applicability is of essence for the development of brain tumor research. Overcoming these treatment induced problems in the coregistration makes our semi-automatic coregistration a valuable method to facilitate research in the expanding area of personalized medicine in brain tumor patients. 


\section{CONCLUSION}

We developed a semi-automatic coregistration method for MRI images of brain tumors to allow the accurate evaluation of treatment response in further research. We have demonstrated show good performance of this approach using 3D structural similarity and targeted registration error methods. We have also provided a detailed description of methodology using freely available software, making it reproducible by the neuroimaging community. This is an essential tool for the growing research area of brain tumor imaging and treatment response evaluation in large sets of patients.

\section{ACKNOWLEDGMENTS}

This research was funded by a National Institute of Health Clinician Scientist Fellowship [SJP], a Remmert Adriaan Laan Fund [AH], a René Vogels Fund [AH] and a grant from the Chang Gung Medical Foundation and Chang Gung Memorial Hospital, Keelung [JLY]. None of the authors have financial of other conflict of interest related to the work presented in this paper. This paper presents independent research funded by the UK National Institute for Health Research (NIHR). The views expressed are those of the author(s) and not necessarily those of the UK NHS, the UK NIHR or the UK Department of Health.

Formatted: Font: 12 pt

Formatted: Font: $12 \mathrm{pt}$

Formatted: Font: $12 \mathrm{pt}$

Formatted: Font: $12 \mathrm{pt}$

Formatted: Font: $12 \mathrm{pt}$ 


\section{REFERENCES}

1. Roger Stupp, M.D., Warren P. Mason, M.D., Martin J. van den Bent, M.D., Michael Weller, M.D., Barbara Fisher, M.D., Martin J.B. Taphoorn, M.D., Karl Belanger, M.D., Alba A. Brandes, M.D., Christine Marosi, M.D., Ulrich Bogdahn, M.D., Jürgen Curschmann, M.D., Robert C. Janzer, M.D., Samuel K. Ludwin, M.D., Thierry Gorlia, M.Sc., Anouk Allgeier, Ph.D., Denis Lacombe, M.D., J. Gregory Cairncross, M.D., Elizabeth Eisenhauer, M.D., and René O. Mirimanoff, M.D. for the European Organisation for Research and Treatment of Cancer Brain Tumor and Radiotherapy Groups and the National Cancer Institute of Canada Clinical Trials Group. Radiotherapy plus concomitant and adjuvant temozolomide for glioblastoma. N Engl J Med 2005; 352: 987-996.

2. Burnet NG, Jefferies SJ, Benson RJ, Hunt DP, Treasure FP. Years of life lost (YLL) from cancer is an important measure of population burden and should be considered when allocating research funds. J Cancer 2005; 92: 241-245.

3. Cuddapah VA, Robel S, Watkins S, Sontheimer H. A neurocentric perspective on glioma invasion. Nat Rev Neurosci 2014; 15: 455-465.

4. Nabavi A, Black PM, Gering DT, Westin CF, Mehta V, Pergolizzi RS Jr, Ferrant M, Warfield SK, Hata N, Schwartz RB, Wells WM 3rd, Kikinis R, Jolesz FA. Serial Intraoperative MR imaging of brain shift. Neurosurgery 2001; 48: 787-797.

5. Shibamoto Y, Baba F, Oda K, Hayashi S, Kokubo M, Ishihara S, Itoh Y, Ogino H, Koizumi M. Incidence of brain atrophy and decline in mini-mental state examination score after whole-brain radiotherapy in patients with brain metastases: A prospective study. Int J Radiat Oncol Biol Phys 2008; 72 : 1168-1173. 
6. Jenkinson $M$, Bannister $P$, Brady $M$, Smith $S$. Improved optimization for the robust and accurate linear registration and motion correction of brain images. Neuroimage 2002; 17: 825-841.

7. Klein A, Andersson J, Ardekani BA, Ashburner J, Avants $\quad B$, Chiang MC, Christensen GE, Collins DL, Gee J, Hellier P, Song JH, Jenkinson M, Lepage C, Rueckert D, Thompson P, Vercauteren T, Woods RP, Mann JJ, Parsey RV. Evaluation of 14 nonlinear deformation algorithms applied to human brain MRI registration. Neuroimage 2009; 46: 786-802.

8. Zacharaki El, Hogea CS, Shen D, Biros G, Davatzikos C. Non-diffeomorphic registration of brain tumor images by simulating tissue loss and tumor growth. Neuroimage 2009; 46: 762-774.

9. Mohamed A, Zacharaki El, Shen D, Davatzikos C. Deformable registration of brain tumor images via a statistical model of tumor-induced deformation. Med Image Anal 2006; 10: 752-763.

10. Ellingson BM, Cloushesy TF, Lai A, Nghiemphu PL, Pope WB. Nonlinear registration of diffusion-weighted images improves clinical sensitivity of functional diffusion maps in recurrent glioblastoma treated with bevacizumab. Magn Reson Med 2012; 67: 237-245.

11. Kaal ECA, Niël CGJH, Vecht CJ. Therapeutic management of brain metastasis. Lancet Neurol 2005; 4: 289-298.

12. Whittle IR. Surgery for gliomas. Curr Opin Neurol 2002; 15: 663-669.

13. Hiramatsu R, Kawabata S, Furuse M, Miyatake SI, Kuroiwa T. Identification of early and distinct glioblastoma response patterns treated by boron neutron capture therapy not predicted by standard radiographic assessment using functional diffusion map. Radiat Oncol 2013; 8: 192. 
14. Moffat BA, Chenevert TL, Lawrence TS, Myer CR, Johnson TD, Dong Q, Tsien C, Mukherji S, Quint DJ, Gebarski SS, Robertson PL, Junck LR, Rehemtulla A, Ross BD. Functional diffusion map: A noninvasive MRI biomarker for early stratification of clinical brain tumor response. Proc Natl Acad Sci USA 2005; 102 : 5524-5529.

15. Tsien C, Galbán CJ, Chenevert TL, Johnson TD, Hamstra DA, Sundgren PC, Junck L, Meyer CR, Rehemtulla A, Lawrence T, Ross BD. Parametric response map as an imaging biomarker to distinguish progression from pseudoprogression in high-grade glioma. J Clin Oncol 2010; 28: 2293-399.

16. Chitphakdithai N, Chian VL, Duncan JS. Non-rigid registration of longitudinal brain tumor treatment MRI. Conf Pric IEEE Eng Med Biol Soc 2011; 2011: 4893-4896.

17. Chitphakdithai N, Vives KP, Duncan JS. Registration of brain resection MRI with intensity and location priors. Proceedings of the IEEE International Symposium on Biomedical Imaging: From Nano to Macro, Chicago, USA, 2011; 1520-1523.

18. Liu Y, Yao C, Zhou LF, Chrisochoides N. A point based non-rigid registration for tumor resection using IMRI. Proceddings of the IEEE International Symposium on Biomedical Imaging: From Nano to Macro, Rotterdam, the Netherlands, 2010; 1217-1220.

19. Nithiananthan S, Schafer S, Mirota DJ, Stayman JW, Zbijewski W, Reh DD, Gallia GL, Siewerdsen JH. Extra-dimensional Demons: A method for incorporating missing tissue in deformable image registration. Med Phys 2012; 39: $5718-5731$.

20. Ding S, Miga M, Noble JH, Cao NA, Dumpuri P, Thompson RC, Dawant BM. Semiautomatic registration of pre- and postbrain tumor resection laser range data: Methods and validation. IEEE Trans Biomed Eng 2009; 56: 770-780. 
21. Rivaz H, Chen SJS, Collins DL. Automatic deformable MR-ultrasound registration for image-guided neurosurgery. IEEE Trans Med Imaging 2015; 34: 366-380.

22. Risholm P, Samset E, Talos IF, Wells W. A non-rigid registration framework that accommodates resection and retraction. Inf Process Med Imaging 2009; 21: 447-458.

23. Smith SM. Fast robust automated brain extraction. Hum Brain Mapp 2002; 17 : 143-155.

24. Zhang $Y$, Brady M, Smith S. Segmentation of brain MR images through a hidden markov random field model and the expectation-maximization algorithm. IEEE Trans Med Imaging 2001; 20: 45-57.

25. Wang Z, Bovik AC, Sheikh HR, Simoncelli EP. Image qualifty assessment: From error visibility to structural similarity. IEEE Trans Image Process 2004; 13: $600-612$.

26. Cohen DS, Lustgarten JH, Miller E, Khandji AG, Goodman RR. Effects of coregistration of MR to CT images on MR stereotactic accuracy. J. Neurosurg 1995; 82: 772-779.

27. Schucht P, Knittel S, Slotboom J, Seidel K, Murek M, Jilch A, Raabe A, BeckJ. 5-ALA complete resections go beyond MR contrast enhancement: shift corrected volumetric analysis of the extent of resection in surgery for glioblastoma. Acta Neurochi 2014; 156: 305-312 
6 7 8 9

TABLE 1 - Targeted coregistration errors between pre- and post-operation

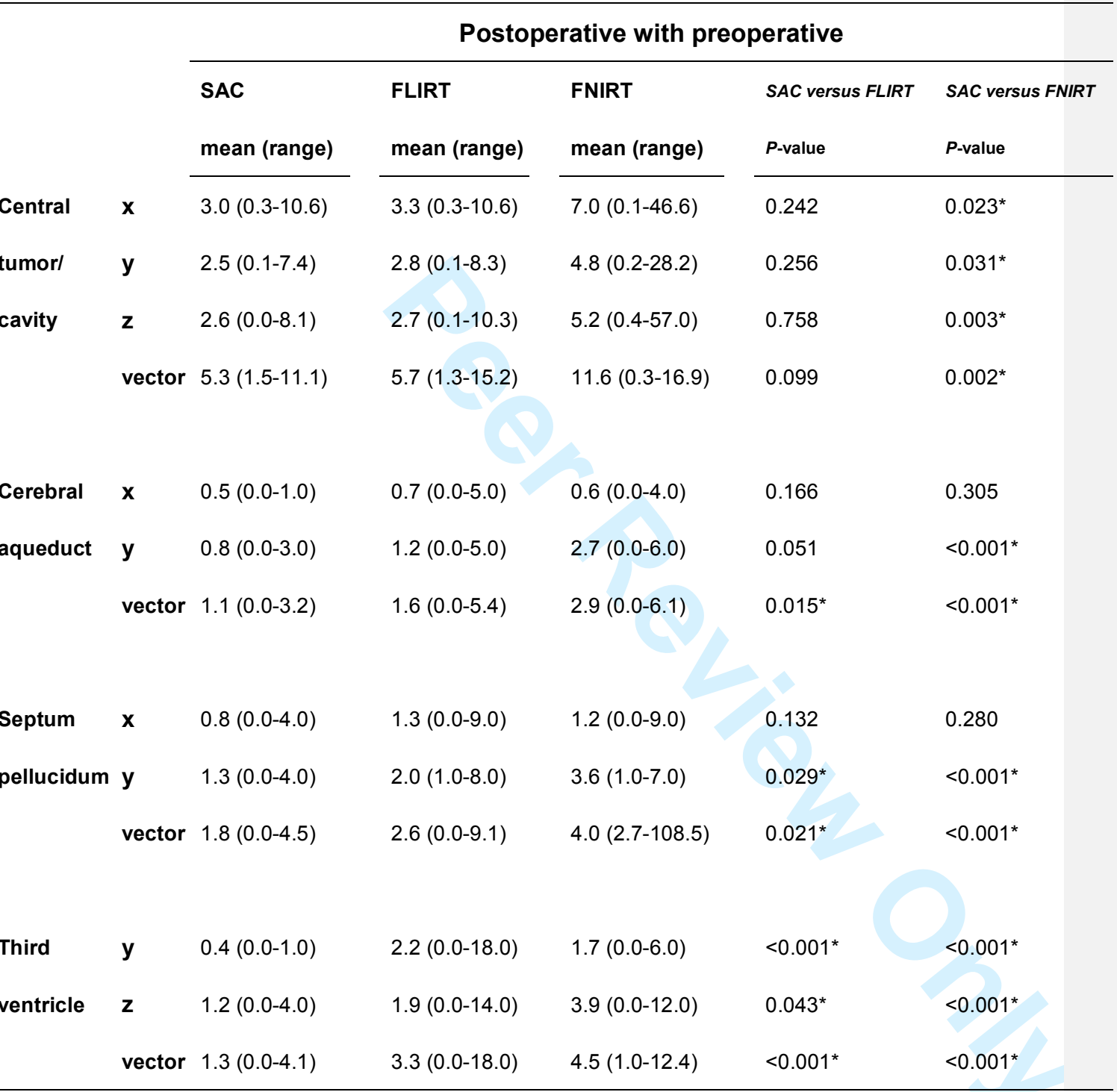

Targeted registration errors are provided with the deviations $(\mathrm{mm})$ of the anatomical landmark coordinates from the reference images after coregistration of the postoperative to the preoperative scan and the preoperative with the recurrence scan. The semi-automatic non-linear coregistration (SAC), linear (FLIRT) and default non-linear (FNIRT) coregistration are compared. An * indicates statistical significance. 


\section{TABLE 2 - Targeted coregistration errors between pre-operation and}

recurrence

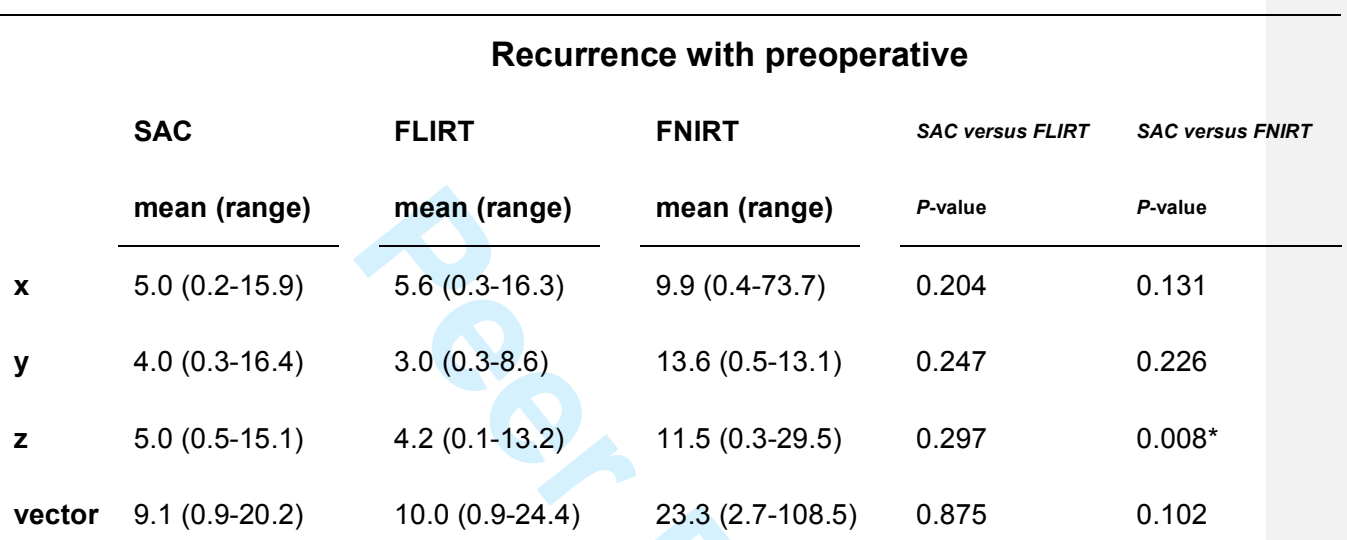

$\begin{array}{lllllll}\text { Septum } & \mathbf{x} & 2.8(0.0-12.0) & 3.3(0.0-14.0) & 2.0(0.0-19.0) & 0.2132 & 0.188 \\ \text { pellucidum } & \mathbf{y} & 2.8(0.0-15.0) & 2.8(0.0-16.0) & 3.0(0.0-17.0) & 1.0000 & 0.779 \\ & \text { vector } & 4.3(1.0-19.0) & 4.6(0.0-20.6) & 4.1(0.0-10.2) & 0.7280 & 0.782 \\ & & & & & & \\ & & & & & & \\ \text { Third } & \mathbf{y} & 0.6(0.0-3.0) & 3.0(0.0-16.0) & 3.1(0.0-11.0) & 0.0010^{*} & 0.001^{*} \\ \text { ventricle } & \mathbf{z} & 2.6(0.0-9.0) & 2.5(0.0-13.0) & 3.1(0.0-16.0) & 0.8898 & 0.466 \\ & \text { vector } & 2.7(0.0-9.1) & 4.6(1.0-16.0) & 5.1(0.0-16.5) & 0.0112^{*} & 0.006^{*}\end{array}$

$\begin{array}{llll}1.3(0.0-4.0) & 0.8(0.0-6.0) & 0.822 & 0.280 \\ 2.1(0.0-5.0) & 2.3(0.0-8.0) & 0.004^{*} & 0.032^{*} \\ 2.8(0.0-5.0) & 2.7(0.0-8.2) & 0.016^{*} & 0.124\end{array}$

Targeted registration errors are provided with the deviations $(\mathrm{mm})$ of the anatomical landmarks coordinates from the reference images after coregistration of the recurrence to the preoperative scan and the preoperative with the recurrence scan. The semi-automatic non-linear coregistration (SAC), linear (FLIRT) and default non-linear (FNIRT) coregistration are compared. An * indicates statistical significance. 


\title{
Validation of a semi-automatic coregistration of MRI scans in brain tumor patients during treatment follow-up
}

\author{
Anouk van der Hoorn, MD PhD ${ }^{1-3+}$; Jiun-Lin Yan, $\mathrm{MD}^{1,4-5 \dagger^{*}}$; Timothy J Larkin, $\mathrm{PhD}^{1}$; \\ Natalie R Boonzaier, MSc ${ }^{1}$, Tomasz Matys, MD PhD²; Stephen J Price, BSc \\ MBBS(Hons) PhD FRCS(Neuro Surg. $)^{1}$ \\ ${ }^{\dagger}$ contributed equally; \\ ${ }^{1}$ Brain tumor imaging lab, Division of neurosurgery, Department of clinical \\ neuroscience, University of Cambridge, Addenbrooke's hospital, Box 167, CB2 0QQ, \\ Cambridge, United Kingdom; \\ 2 Department of radiology, University of Cambridge, Addenbrooke's hospital, Box \\ 218, CB2 0QQ, Cambridge, United Kingdom; \\ ${ }^{3}$ Department of radiology (EB44), University Medical Centre Groningen, University of \\ Groningen, Box 30.001, 9700 RB, Groningen, The Netherlands; \\ ${ }^{4}$ Department of neurosurgery, Chang Gung Memorial Hospital, 22 Majing road, 204, \\ Keelung, Taiwan \\ ${ }^{5}$ Department of neurosurgery, Chang Gung University College of Medicine, 259 \\ Wenhua $1^{\text {st }}$ road, 333, Taoyuan, Taiwan
}

Corresponding author:

Jiun-Lin Yan

Division of neurosurgery

Department of Clinical neuroscience

University of Cambridge

Addenbrooke's hospital

Box 167

CB2 0QQ

Cambridge, United Kingdom

Running title: Coregistration of MRI in brain tumor patients

Word count: 4342

Figures: 5

Tables: 2

Supporting material for online-only publication: Yes 


\begin{abstract}
There is an expanding research interest in high grade gliomas due to their significant population burden and poor survival despite the extensive standard multimodal treatment. One of the obstacles is the lack of individualized monitoring of tumor characteristics and treatment response before, during and after treatment. We developed a two stage semi-automatic method to coregister MRI scans at different time points before and after surgical and adjuvant treatment of high grade gliomas. This two stage coregistration includes a linear coregistration of the semi-automatically derived mask of the preoperative contrast enhancing area or postoperative resection cavity, brain contour, and ventricles between different time points. The resulting transformation matrix was then applied to a nonlinear manner to coregister conventional contrast-enhanced T1-weighted images. Targeted registration errors were calculated and compared to linear and nonlinear coregistered images. Targeted registration errors were smaller for the semi-automatic non-linear coregistration compared with both the nonlinear and linear coregistered images. This was further visualized utilizing a 3D structural similarity method. The semi-automatic non-linear coregistration allowed for optimal correction of variable brain shift at different time points as evaluated by minimal targeted registration error. This proposed method allows for accurate evaluation of treatment response, essential for the growing research area of brain tumor imaging and treatment response evaluation in large sets of patients.
\end{abstract}

Keywords: Linear coregistration; nonlinear coregistration; brain tumors; high grade gliomas; MRI; treatment response; validation; structure similarity; 


\section{Abbreviation:}

2D (two-dimensional)

3D (three-dimensional)

FMRIB (Oxford centre for functional MRI of the brain)

FMRIB Software Library (FSL)

FAST (FMRIB's Automated Segmentation Tool)

FLIRT (FMRIB's linear image registration tool)

FNIRT (FMRIB's non-linear image registration tool)

MNI (Montreal Neurological Institute)

SAC (Semi-automatic non-linear coregistration) 


\section{INTRODUCTION}

Despite advancements in treatment of high grade gliomas, including the introduction of concomitant chemoradiotherapy and adjuvant chemotherapy (1), these tumors continue to carry a high mortality rate and significant population burden (2-3). One of the unmet needs in treatment development research is the ability to easily identify differences in tumor characteristics and treatment response with MRI imaging biomarkers before, during and after therapy. This would facilitate research on treatment response in large sets of patients and discovery of new imaging biomarkers, further enabling personalization of therapy. In order to meet this demand, an easily applicable coregistration method is needed to coregister postoperative with preoperative images during MRI assessment of high grade gliomas.

A nonlinear registration is needed as brain shift and deformation pose significant challenges when comparing brain MR images at different time points. These changes do not only occur after the initial surgical procedure, but are rather a dynamic continuous process (4). In addition to the structural changes caused by surgery, tumor response to treatment and chronic radiotherapy effects induce further changes. These include, for example, changes in tumor volume (5), making the coregistration process even more difficult.

Though previous research has aimed to address these obstacles, an easily accessible and applicable coregistration method is not yet available for preoperative and postoperative MRI scans of patients with brain tumors. Most methods focus on coregistration of different sequences of the same scan time point with linear and nonlinear registration methods in healthy subjects and brain tumor patients. These existing methods demonstrate good performances for this intrasubject coregistration 
of data from the same time (6-7) or to a standardized brain atlas (8-9). Research has also demonstrated the value of a nonlinear coregistration for treatment response evaluation in patients without surgery (10). However, surgery is part of the standard treatment scheme in patients with high grade gliomas (1) and is regularly performed in other primary and metastatic brain tumors (11-12). Therefore, the ability to deal with post-surgical changes should be part of the coregistration method.

Methods of MRI coregistration taking resection into account are scarce and have several limitations making them unsuitable as a widely and easily usable coregistration method. The few available clinical studies that have used an intrasubject coregistration method after surgery are difficult to replicate and evaluate as they use in-house software, and provide only limited details of methodology (e.g. 13-15). Technical studies suffer from other issues, such as small sample size (16). Another study has tested the methods only on epilepsy patients with whole lobe resection (17), where less signal changes and mass effects are expected. Furthermore, these and other technical papers do not provide comprehensible coregistration guideline and thereby hinder wide applicability, especially for clinical researchers (16-18).

There are a few methods available that coregister intraoperative images with preoperative imaging. However, these are limited by using other modalities, like CT (19), tracked laser range scanning (20) or ultrasound (21). CT is inferior to MRI in detecting tumor recurrence and thus not routinely used for the treatment evaluation or in research of brain tumor patients. Ultrasound and laser imaging are only possible during surgery when the skull is temporarily removed. The method from Nithiananthan and colleagues (19) uses an approach that defines resected voxels 
based on an air density. This is not applicable to research with interest in tumor response assessment using postoperative MRI imaging as the resection cavity being filled with fluid and/or adjacent brain tissue. Other preoperative with intraoperative coregistration methods have only tested the complex algorithm on 2D data (22).

Therefore, a need for an easily applicable and usable coregistration method before, during and after treatment including surgical resection is clearly required. To address this, we developed a semi-automatic coregistration technique using widely used and freely available software. This may allow accurate evaluation of treatment response in future studies, which is essential for brain tumor imaging research and treatment response assessment in large sets of patients. We also provide detailed information about the steps and the code used. 


\section{METHODS}

\section{Patient inclusion criteria}

Patients with newly diagnosed supratentorial glioblastoma, operated from 2010 to 2014, were included in the study. Exclusion criteria were previous cranial surgery, previous cerebral radiotherapy or another known primary tumor. We included 32 patients (mean age 56 years, range 31-68; 20 males) with preoperative MRI scans that also had available follow-up MRI. Follow-up MRI scans included a direct postoperative scan and the scan at the time-point of tumor recurrence. Adequate direct postoperative MRI data ( $<72$ hours) was available for 30 patients, while later follow-up at the time of tumor recurrence was available for 27 patients (mean 12 months after the operation date, range 0.8-38). All patients were treated with maximal surgical resection followed by standard concomitant chemoradiotherapy followed by adjuvant chemotherapy (1).

The study was approved by the local Institutional Review Board (10/H0308/23) and informed written consent was obtained from all patients.

\section{Data acquisition}

Preoperative MRI data acquisition was performed using a 3.0 T Siemens Magnetom MRI system (Siemens Healthcare, Munich, Germany) with a standard 12-channel head coil. Imaging included an anatomical 3D T1-weighted sequence with fat suppression acquired after the intravenous injection of $9 \mathrm{~mL}$ gadolinium (Gadovist, Bayer Schering Pharma, Berlin, Germany) (TR/TE 900/2.98 ms, inversion time 900 ms; flip angle 9, FOV $256 \times 240$ mm; 176-208 slices; no slice gap; voxel size $1 \times 1 \times$ $1 \mathrm{~mm})$. 
Follow-up MRI scans were acquired on a 1.5 T GE Optima, 1.5 or 3.0 T GE Signa (General Electric Company, Little Chalfont, United Kingdom) or $1.5 \mathrm{~T}$ Siemens Avanto (Siemens Healthcare, Munich, Germany) with standard head coil. Imaging included a T1-weighted anatomical sequence after the intravenous injection of $9 \mathrm{~mL}$ gadolinium (Gadovist, Bayer Schering Pharma, Berlin, Germany). This was performed as a 2D T1-weighted sequence (TR/TE 440-771/8-21 ms, flip angle 58-90, FOV 220-240 x 207-240 mm; 20-85 slices; 0-1 mm slice gap; voxel size 0.429-0.7188 x 0.429-0.7188 × 3-6 mm), a 2D T1 inversion recovery sequence (TR/TE 2508-2600/12-42 ms, inversion time 780-920 ms; flip angle 90-110 FOV $220 \times 220 \mathrm{~mm} ; 20-22$ slices; $1-3.5 \mathrm{~mm}$ slice gap; voxel size of $0.4297 \times 0.4297 \times 6$ $\mathrm{mm}$ ) or a 3D T1 fat suppressed sequence (TR/TE 7/2.948 ms, inversion time $900 \mathrm{~ms}$; flip angle $190^{\circ}$, FOV $256 \times 256$ mm; 190 slices; no slice gap; voxel size 1 x 1 x $1 \mathrm{~mm}$ ).

\section{Coregistration method}

Coregistration was performed using a two-staged semi-automatic method (Figure 1). Conventional contrast-enhanced T1-weighted images were coregistered using tools from the FMRIB Software Library (FSL) version 5.0 .0 (http://fsl.fmrib.ox.ac.uk/fsl/fslwiki/). Before the coregistration stages commence, all images were realigned with the anterior commissure as the center point (coordinate 0 , $0,0)$ to minimize the influence of brain position. The first stage was the coregistration of the binary masks, which consisted of the outer contour of the brain, the ventricles, and contrast enhancing area (presurgical MRI images) or resection area (follow up MRI images). This was performed for each subject at the different time points to create a transformation matrix (Figure 1, step 4, using the FLIRT, FMRIB's linear image registration tool, function). The brain contours were created from the inversion of the brain masks which were semi-automatically extracted (23). This was followed by 
manual correction (Figure 1, step 1 and 2) and the resulting mask was binarised. The ventricles were identified with an automatic segmentation using the FSL FAST (Figure1, step 3, FMRIB's automated segmentation tool) function (24). The FAST function also allowed a semi-automatic identification of the contrast enhancing area or resection cavity. The contrast enhancing area is targeted for resection and is replaced by the resection cavity on the direct postoperative and later follow-up MRI scans. Therefore, this stage of coregistration allowed for optimal correction of variable brain shift and surgery-induced changes at different time points.

The second stage applied the transformation matrix, acquired from the first stage, as input for a nonlinear transformation matrix of the brain images (Figure 1, step 5 and 6, using the FNIRT, FMRIB's non-linear image registration tool, functions). The nonlinear transformation of the brain images used additional subsampling levels for regularization. This also included the binary masks of the brain contour and ventricles from both the preoperative reference image and the follow-up image. This resulted in a coregistration of the follow-up brain extracted MRI scans with the preoperative brain extracted MRI scans. The steps with accompanying FSL code are illustrated (Figure 1) and are provided as supporting material to facilitate use by others (see supporting material). Standard linear coregistration (FLIRT function) and standard FNIRT with default setting were done separately for comparison using the FLIRT and FNIRT option in FSL.

\section{Validation methods}

Validation was performed using a targeted registration error method for calculating the error in different directions. Validation in the $\mathrm{x}$ and $\mathrm{y}$ directions was performed utilizing the cerebral aqueduct and septum pellucidum on the same axial slice (Figure 
2A). Validating in the $y$ and $z$ directions (Figure 2B) was performed utilizing the upper anterior boundary of the third ventricle, at the level of the foramen of Monro (Figure $2 \mathrm{C})$, on the same coronal slice. The central point of the tumor or cavity was targeted automatically for the calculation of the registration error at the location where most errors could be expected. Vectors were also calculated for all targets. All targeted registration errors were calculated for the semi-automatic non-linear coregistration method and compared to the targeted registration errors for the linear coregistered and standard non-linear coregistered images. Differences were tested with a Wilcoxon signed rank test or paired t-test depending on the normality. Two-sided p-values were used.

In addition, a 3D structural similarity map was created (25) using Matlab (MathWorks Inc., Natick, MA, USA). The 3D structural similarity map was created for each subject comparing the preoperative reference scan with the coregistered follow-up scan. This was done for scans obtained direct postoperative and at tumor recurrence separately. To display group results, the preoperative images were coregistered to standard MNI (Montreal Neurological Institute) space with a nonlinear transformation of the brain images including a lesion mask. This was preceded by a linear transformation of a binary mask of the brain exterior and ventricles. A mean structural similarity mask was then created by transforming the structural similarity mask of each patient to standard MNI space. The resection cavity or contrast enhancing areas were excluded for the mean structural similarity image, as these values are inherently different. One author (JLY) also visually inspected all the coregistered MRI scans for accuracy. 


\section{RESULTS}

\section{Targeted registration error}

The targeted registration error showed good performance of the coregistration method for the direct postoperative and recurrence images with a clear benefit over the linear coregistration method and standard FNIRT non-linear coregistration method (Table 1). In the coregistration of postoperative to preoperative images, in comparison to the FLIRT, the SAC non-linear coregistration showed a smaller vector deviation of the cerebral aqueduct (1.1 versus $1.6, p=0.015)$. A smaller deviation was also detected for the septum pallucidum y coordinate and vector (1.3 versus 2.0, $p=0.029 ; 1.8$ versus $2.6, p=0.021$ ), as well as the upper most of third ventricle $y, z$ coordinate and vector ( 0.4 versus $2.2, p<0.001 ; 1.2$ versus $1.9, p=0.043 ; 1.3$ versus 3.3, $p<0.001)$. The semi-automatic nonlinear coregistration also outperformed the default FNIRT coregistration, where there was small deviation between preoperative and postoperative images for most of the coordinates and vectors in the central tumor/cavity point, cerebral aqueduct, septum pellucidum and the third ventricle at the level of foramen of Monro.

The benefit of this coregistration method can also be seen in the coregistration of recurrent images to preoperative images (Table 2). The target error at the cerebral aqueduct and the roof of the third ventricle at the level of foramen of Monro was smaller using the semi-automatic non-linear coregistration than the linear coregistration and FNIRT coregistration. When using default FNIRT, there was a larger $z$ coordinate deviation at the tumor centroid point. Additionally, the linear coregistration failed in three patients, and default FNIRT coregistration failed in 2 patients, for both the postoperative and recurrence scan, while the semi-automatic method was used without problems. 


\section{D structural similarity}

Mean 3D structural similarity of all 32 patients showed the relative performance of the coregistration method (Figure 3), which is also illustrated using a representative subject using contour overlay images (Figure 4). The peripheral areas, including the frontal and parietal areas, demonstrated the best performance. A good performance was also seen at the periventricular regions. A relatively lower overlap between the coregistered and reference preoperative scan was seen in the midsagittal and central areas, the centrum semiovale and central cerebellum.

An example of this coregistration is shown in figure 5. Without proper coregistration, one cannot confidently compare the initial postoperative or tumour recurrence images with the pre-operative reference images (Figure 5A). Although the standard FNIRT coregistration of postoperative MR and recurrent MR coregistration (Figure 5B) realigned images in the same space as the reference images, regional torsions (white arrows) were detected at the resection edges. By using the semi-automatic coregistration methods, the coregistered postoperative MR (Figure 5C left) and the recurrent MR (Figure 5C middle) were able to show the residual contrast enhanced lesion (yellow contour) and the recurrent area (red contour) in the reference MR.(Figure 5C). 


\section{DISCUSSION}

We developed and validated a two staged semi-automatic method for the coregistration of preoperative and follow-up MRI scans for both the direct postoperative and tumor recurrence time point (Table1, Table 2, and Figure 5). The semi-automatic derived mask of the outer brain contour, ventricles and lesion allowed an accurate coregistration despite the changes in brain shift and postoperative changes. Therefore, this method is highly applicable to analyze large imaging datasets to evaluate treatment response, a growing and clinically important research area. Furthermore, it can be easily reproduced, allowing a wide applicability of the method.

In the standard FNIRT function, an affine transformation is required as a starting guess of the coregistration. This affine transformation is typically the result of linear FLIRT coregistration between different MR series. However, the deformation in different time points happens mainly over the peritumoral regions, resection cavities, and ventricles. Our approach was to use the transformation affine of these areas with the greatest changes rather than the whole brain images (Figure 1 step 4). Furthermore, we added an inverted brain mask as an outer frame to our first stage coregistration for the gross spatial position (Figure 1 step 2 and 3 ). This resulting affine was further applied to the normal brain parenchyma with the mask of ventricle and lesion (Figure1 step 5) to achieve the optimal coregistration.

To the best of our knowledge, there is a shortage of easily applicable methods that allow coregistration of preoperative and postoperative imaging in patients with brain tumors. Clinical papers often use in-house software solutions and provide only partial description of the methodology (e.g. 13-15). This hinders reproducibility and makes 
independent evaluation of reliability difficult. On the other hand, technical papers are often complex and use only a small number of patient datasets, up to two patients with brain tumors (16) and up to six patients with epilepsy surgery (17). We created an easily reproducible method (see online-only material for the code) and, tested it in a large $(N=32)$ population. Previous studies utilizing intraoperative coregistration have shown a clear advantage of nonlinear coregistration with high diagnostic and stereotactic accuracy $(10,26)$. This supports the results of the postoperative coregistrations from our study.

This two-staged semi-automatic coregistration method can be replicated by using the FMRIB Software Library which is a freely available and widely used software package in the neuroimaging research community. We have provided a detailed description of the steps required to recapitulate this approach (see Figure 1 and supporting material). This makes this method easily reproducible by others, including clinical researchers, which further supported the rationale of using FSL for our coregistration method. Given this purpose of this study, we have not tried to validate the coregistration of patients with brain tumors using other software packages. Interpolation of our semi-automatic method to other software packages might be possible, but would require separate validation.

The MR image acquisition parameters in our dataset varied across subjects and time points. In particular, a contrast-enhanced 3D T1-weighted dataset was available for the preoperative scan, but the follow-up images were almost always $2 \mathrm{D}$ and obtained with different MRI parameters and scanners from different manufacturers. Another limitation is that this method assumes that the resection cavity is a result of surgical resection of contrast enhancing tumour only, and that the recurrent tumour arises 
Page 37 of 52

NMR in Biomedicine - For Peer Review Only

1
2
3
4
5
6
7
8
9
10
11
12
13
14
15
16
17
18
19
20
21
22
23
24
25
26
27
28
29
30
31
32
33
34
35
36
37
38
39
40
41
42
43
44
45
46
47
48
49
50
51
52
53
54
55
56
57
58
59
60

from non-contrast enhancing surrounding area. However, the resection volume may extend beyond the area of contrast enhancement to include the 'peri-tumoural region', particularly if the resection is conducted under the fluorescence guidance of 5 aminoleuvulinic acid (27). Despite these differences, our validation showed that the method worked in all subjects, making it easily applicable in clinical practice.

At a time when adequate imaging biomarkers are being sought to evaluate treatment response, method applicability is of essence for the development of brain tumor research. Overcoming these treatment induced problems in the coregistration makes our semiautomatic coregistration a valuable method to facilitate research in the expanding area of personalized medicine in brain tumor patients.

http://mc.manuscriptcentral.com/nbm 


\section{CONCLUSION}

We developed a semi-automatic coregistration method for MRI images of brain tumors to allow the accurate evaluation of treatment response in further research. We have demonstrated show good performance of this approach using 3D structural similarity and targeted registration error methods. We have also provided a detailed description of methodology using freely available software, making it reproducible by the neuroimaging community. This is an essential tool for the growing research area of brain tumor imaging and treatment response evaluation in large sets of patients.

\section{ACKNOWLEDGMENTS}

This research was funded by a National Institute of Health Clinician Scientist Fellowship [SJP], a Remmert Adriaan Laan Fund $[\mathrm{AH}]$, a René Vogels Fund $[\mathrm{AH}]$ and a grant from the Chang Gung Medical Foundation and Chang Gung Memorial Hospital, Keelung [JLY]. None of the authors have financial of other conflict of interest related to the work presented in this paper. This paper presents independent research funded by the UK National Institute for Health Research (NIHR). The views expressed are those of the author(s) and not necessarily those of the UK NHS, the UK NIHR or the UK Department of Health. 


\section{REFERENCES}

1. Roger Stupp, M.D., Warren P. Mason, M.D., Martin J. van den Bent, M.D., Michael Weller, M.D., Barbara Fisher, M.D., Martin J.B. Taphoorn, M.D., Karl Belanger, M.D., Alba A. Brandes, M.D., Christine Marosi, M.D., Ulrich Bogdahn, M.D., Jürgen Curschmann, M.D., Robert C. Janzer, M.D., Samuel K. Ludwin, M.D., Thierry Gorlia, M.Sc., Anouk Allgeier, Ph.D., Denis Lacombe, M.D., J. Gregory Cairncross, M.D., Elizabeth Eisenhauer, M.D., and René O. Mirimanoff, M.D. for the European Organisation for Research and Treatment of Cancer Brain Tumor and Radiotherapy Groups and the National Cancer Institute of Canada Clinical Trials Group. Radiotherapy plus concomitant and adjuvant temozolomide for glioblastoma. N Engl J Med 2005; 352: 987-996.

2. Burnet NG, Jefferies SJ, Benson RJ, Hunt DP, Treasure FP. Years of life lost (YLL) from cancer is an important measure of population burden and should be considered when allocating research funds. J Cancer 2005; 92: 241-245.

3. Cuddapah VA, Robel S, Watkins S, Sontheimer H. A neurocentric perspective on glioma invasion. Nat Rev Neurosci 2014; 15: 455-465.

4. Nabavi A, Black PM, Gering DT, Westin CF, Mehta V, Pergolizzi RS Jr, Ferrant M, Warfield SK, Hata N, Schwartz RB, Wells WM 3rd, Kikinis R, Jolesz FA. Serial Intraoperative MR imaging of brain shift. Neurosurgery 2001; 48: 787-797.

5. Shibamoto Y, Baba F, Oda K, Hayashi S, Kokubo M, Ishihara S, Itoh Y, Ogino H, Koizumi M. Incidence of brain atrophy and decline in mini-mental state examination score after whole-brain radiotherapy in patients with brain metastases: A prospective study. Int J Radiat Oncol Biol Phys 2008; 72: 1168-1173. 
6. Jenkinson $M$, Bannister $\mathrm{P}$, Brady $\mathrm{M}$, Smith $\mathrm{S}$. Improved optimization for the robust and accurate linear registration and motion correction of brain images. Neuroimage 2002; 17: 825-841.

7. Klein A, Andersson J, Ardekani BA, Ashburner J, Avants B, Chiang MC, Christensen GE, Collins DL, Gee J, Hellier P, Song JH, Jenkinson M, Lepage C, Rueckert D, Thompson P, Vercauteren T, Woods RP, Mann JJ, Parsey RV. Evaluation of 14 nonlinear deformation algorithms applied to human brain MRI registration. Neuroimage 2009; 46: 786-802.

8. Zacharaki El, Hogea CS, Shen D, Biros G, Davatzikos C. Non-diffeomorphic registration of brain tumor images by simulating tissue loss and tumor growth. Neuroimage 2009; 46: 762-774.

9. Mohamed A, Zacharaki El, Shen D, Davatzikos C. Deformable registration of brain tumor images via a statistical model of tumor-induced deformation. Med Image Anal 2006; 10: 752-763.

10. Ellingson BM, Cloushesy TF, Lai A, Nghiemphu PL, Pope WB. Nonlinear registration of diffusion-weighted images improves clinical sensitivity of functional diffusion maps in recurrent glioblastoma treated with bevacizumab. Magn Reson Med 2012; 67: 237-245.

11. Kaal ECA, Niël CGJH, Vecht CJ. Therapeutic management of brain metastasis. Lancet Neurol 2005; 4: 289-298.

12. Whittle IR. Surgery for gliomas. Curr Opin Neurol 2002; 15: 663-669.

13. Hiramatsu R, Kawabata S, Furuse M, Miyatake SI, Kuroiwa T. Identification of early and distinct glioblastoma response patterns treated by boron neutron capture therapy not predicted by standard radiographic assessment using functional diffusion map. Radiat Oncol 2013; 8: 192. 
14. Moffat BA, Chenevert TL, Lawrence TS, Myer CR, Johnson TD, Dong Q, Tsien C, Mukherji S, Quint DJ, Gebarski SS, Robertson PL, Junck LR, Rehemtulla A, Ross BD. Functional diffusion map: A noninvasive MRI biomarker for early stratification of clinical brain tumor response. Proc Natl Acad Sci USA 2005; 102 : 5524-5529.

15. Tsien C, Galbán CJ, Chenevert TL, Johnson TD, Hamstra DA, Sundgren PC, Junck L, Meyer CR, Rehemtulla A, Lawrence T, Ross BD. Parametric response map as an imaging biomarker to distinguish progression from pseudoprogression in high-grade glioma. J Clin Oncol 2010; 28: 2293-399.

16. Chitphakdithai N, Chian VL, Duncan JS. Non-rigid registration of longitudinal brain tumor treatment MRI. Conf Pric IEEE Eng Med Biol Soc 2011; 2011: 4893-4896.

17. Chitphakdithai N, Vives KP, Duncan JS. Registration of brain resection MRI with intensity and location priors. Proceedings of the IEEE International Symposium on Biomedical Imaging: From Nano to Macro, Chicago, USA, 2011; 1520-1523.

18. Liu Y, Yao C, Zhou LF, Chrisochoides N. A point based non-rigid registration for tumor resection using IMRI. Proceddings of the IEEE International Symposium on Biomedical Imaging: From Nano to Macro, Rotterdam, the Netherlands, 2010; $1217-1220$.

19. Nithiananthan S, Schafer S, Mirota DJ, Stayman JW, Zbijewski W, Reh DD, Gallia GL, Siewerdsen JH. Extra-dimensional Demons: A method for incorporating missing tissue in deformable image registration. Med Phys 2012; 39: 5718-5731.

20. Ding S, Miga M, Noble JH, Cao NA, Dumpuri P, Thompson RC, Dawant BM. Semiautomatic registration of pre- and postbrain tumor resection laser range data: Methods and validation. IEEE Trans Biomed Eng 2009; 56: 770-780. 
21. Rivaz H, Chen SJS, Collins DL. Automatic deformable MR-ultrasound registration for image-guided neurosurgery. IEEE Trans Med Imaging 2015; 34: 366-380.

22. Risholm P, Samset E, Talos IF, Wells W. A non-rigid registration framework that accommodates resection and retraction. Inf Process Med Imaging 2009; 21: 447-458.

23. Smith SM. Fast robust automated brain extraction. Hum Brain Mapp 2002; 17 : 143-155.

24. Zhang $Y$, Brady M, Smith S. Segmentation of brain MR images through a hidden markov random field model and the expectation-maximization algorithm. IEEE Trans Med Imaging 2001; 20: 45-57.

25. Wang Z, Bovik AC, Sheikh HR, Simoncelli EP. Image qualifty assessment: From error visibility to structural similarity. IEEE Trans Image Process 2004; 13: 600-612.

26. Cohen DS, Lustgarten JH, Miller E, Khandji AG, Goodman RR. Effects of coregistration of MR to CT images on MR stereotactic accuracy. J. Neurosurg 1995; 82: 772-779.

27. Schucht P, Knittel S, Slotboom J, Seidel K, Murek M, Jilch A, Raabe A, BeckJ. 5-ALA complete resections go beyond MR contrast enhancement: shift corrected volumetric analysis of the extent of resection in surgery for glioblastoma. Acta Neurochi 2014; 156: 305-312 
TABLE 1 - Targeted coregistration errors between pre- and post-operation

\begin{tabular}{|c|c|c|c|c|c|c|}
\hline & & \multicolumn{5}{|c|}{ Postoperative with preoperative } \\
\hline & & SAC & FLIRT & FNIRT & SAC versus FLIRT & SAC versus FNIRT \\
\hline & & mean (range) & mean (range) & mean (range) & $P$-value & $P$-value \\
\hline Central & $\mathbf{x}$ & $3.0(0.3-10.6)$ & $3.3(0.3-10.6)$ & $7.0(0.1-46.6)$ & 0.242 & $0.023^{*}$ \\
\hline tumorl & $\mathbf{y}$ & $2.5(0.1-7.4)$ & $2.8(0.1-8.3)$ & $4.8(0.2-28.2)$ & 0.256 & $0.031^{*}$ \\
\hline \multirow[t]{2}{*}{ cavity } & $\mathbf{z}$ & $2.6(0.0-8.1)$ & $2.7(0.1-10.3)$ & $5.2(0.4-57.0)$ & 0.758 & $0.003^{*}$ \\
\hline & vector & $5.3(1.5-11.1)$ & $5.7(1.3-15.2)$ & $11.6(0.3-16.9)$ & 0.099 & $0.002^{*}$ \\
\hline Cerebral & $\mathbf{x}$ & $0.5(0.0-1.0)$ & $0.7(0.0-5.0)$ & $0.6(0.0-4.0)$ & 0.166 & 0.305 \\
\hline \multirow[t]{2}{*}{ aqueduct } & $\mathbf{y}$ & $0.8(0.0-3.0)$ & $1.2(0.0-5.0)$ & $2.7(0.0-6.0)$ & 0.051 & $<0.001^{*}$ \\
\hline & vector & $1.1(0.0-3.2)$ & $1.6(0.0-5.4)$ & $2.9(0.0-6.1)$ & $0.015^{*}$ & $<0.001^{*}$ \\
\hline Septum & $\mathbf{x}$ & $0.8(0.0-4.0)$ & $1.3(0.0-9.0)$ & $1.2(0.0-9.0)$ & 0.132 & 0.280 \\
\hline \multirow[t]{2}{*}{ pellucidum } & $y$ & $1.3(0.0-4.0)$ & $2.0(1.0-8.0)$ & $3.6(1.0-7.0)$ & $0.029^{*}$ & $<0.001^{*}$ \\
\hline & vector & $1.8(0.0-4.5)$ & $2.6(0.0-9.1)$ & $4.0(2.7-108.5)$ & $0.021^{*}$ & $<0.001^{*}$ \\
\hline Third & $\mathbf{y}$ & $0.4(0.0-1.0)$ & $2.2(0.0-18.0)$ & $1.7(0.0-6.0)$ & $<0.001^{*}$ & $<0.001^{*}$ \\
\hline \multirow[t]{2}{*}{ ventricle } & $\mathbf{z}$ & $1.2(0.0-4.0)$ & $1.9(0.0-14.0)$ & $3.9(0.0-12.0)$ & $0.043^{*}$ & $<0.001^{*}$ \\
\hline & vector & $1.3(0.0-4.1)$ & $3.3(0.0-18.0)$ & $4.5(1.0-12.4)$ & $<0.001^{*}$ & $<0.001^{*}$ \\
\hline
\end{tabular}

Targeted registration errors are provided with the deviations $(\mathrm{mm})$ of the anatomical landmark coordinates from the reference images after coregistration of the postoperative to the preoperative scan and the preoperative with the recurrence scan. The semi-automatic non-linear coregistration (SAC), linear (FLIRT) and default non-linear (FNIRT) coregistration are compared. An * indicates statistical significance. 
TABLE 2 - Targeted coregistration errors between pre-operation and recurrence

\begin{tabular}{|c|c|c|c|c|c|c|}
\hline & & \multicolumn{5}{|c|}{ Recurrence with preoperative } \\
\hline & & SAC & FLIRT & FNIRT & SAC versus FLIRT & SAC versus FNIRT \\
\hline & & mean (range) & mean (range) & mean (range) & $P$-value & $P$-value \\
\hline Central & $\mathbf{x}$ & $5.0(0.2-15.9)$ & $5.6(0.3-16.3)$ & $9.9(0.4-73.7)$ & 0.204 & 0.131 \\
\hline tumorl & y & $4.0(0.3-16.4)$ & $3.0(0.3-8.6)$ & $13.6(0.5-13.1)$ & 0.247 & 0.226 \\
\hline \multirow[t]{2}{*}{ cavity } & $\mathbf{z}$ & $5.0(0.5-15.1)$ & $4.2(0.1-13.2)$ & $11.5(0.3-29.5)$ & 0.297 & $0.008^{*}$ \\
\hline & vector & $9.1(0.9-20.2)$ & $10.0(0.9-24.4)$ & $23.3(2.7-108.5)$ & 0.875 & 0.102 \\
\hline Cerebral & $\mathbf{x}$ & $1.2(0.0-5.0)$ & $1.3(0.0-4.0)$ & $0.8(0.0-6.0)$ & 0.822 & 0.280 \\
\hline \multirow[t]{2}{*}{ aqueduct } & y & $1.3(0.0-3.0)$ & $2.1(0.0-5.0)$ & $2.3(0.0-8.0)$ & $0.004^{*}$ & $0.032^{*}$ \\
\hline & vector & $2.0(0.0-5.8)$ & $2.8(0.0-5.0)$ & $2.7(0.0-8.2)$ & $0.016^{*}$ & 0.124 \\
\hline Septum & $\mathbf{x}$ & $2.8(0.0-12.0)$ & $3.3(0.0-14.0)$ & $2.0(0.0-19.0)$ & 0.2132 & 0.188 \\
\hline \multirow[t]{2}{*}{ pellucidum } & $y$ & $2.8(0.0-15.0)$ & $2.8(0.0-16.0)$ & $3.0(0.0-17.0)$ & 1.0000 & 0.779 \\
\hline & vector & $4.3(1.0-19.0)$ & $4.6(0.0-20.6)$ & $4.1(0.0-10.2)$ & 0.7280 & 0.782 \\
\hline Third & $y$ & $0.6(0.0-3.0)$ & $3.0(0.0-16.0)$ & $3.1(0.0-11.0)$ & $0.0010^{*}$ & $0.001^{*}$ \\
\hline \multirow[t]{2}{*}{ ventricle } & $\mathbf{z}$ & $2.6(0.0-9.0)$ & $2.5(0.0-13.0)$ & $3.1(0.0-16.0)$ & 0.8898 & 0.466 \\
\hline & vector & $2.7(0.0-9.1)$ & $4.6(1.0-16.0)$ & $5.1(0.0-16.5)$ & $0.0112^{*}$ & $0.006^{*}$ \\
\hline
\end{tabular}

Targeted registration errors are provided with the deviations $(\mathrm{mm})$ of the anatomical landmarks coordinates from the reference images after coregistration of the recurrence to the preoperative scan and the preoperative with the recurrence scan. The semi-automatic non-linear coregistration (SAC), linear (FLIRT) and default non-linear (FNIRT) coregistration are compared. An * indicates statistical significance. 


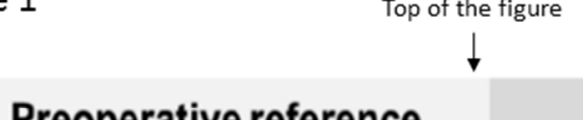

\section{Preoperative reference}
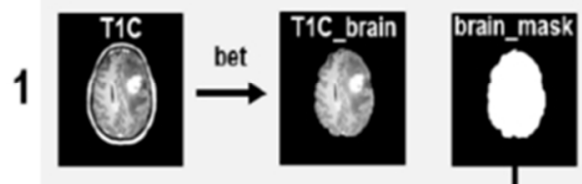

invert mask

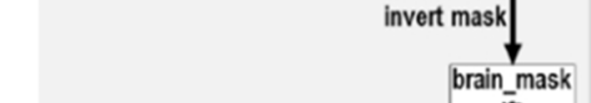

2
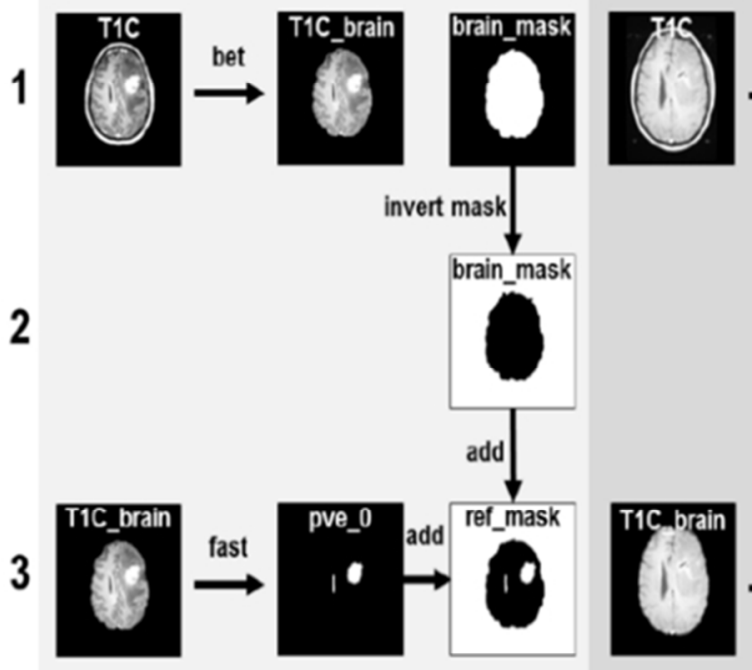

\section{Follow-up input}

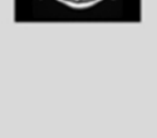

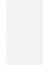

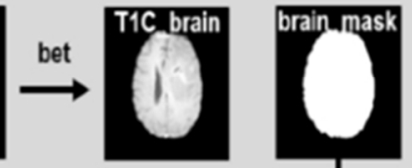

invert mask
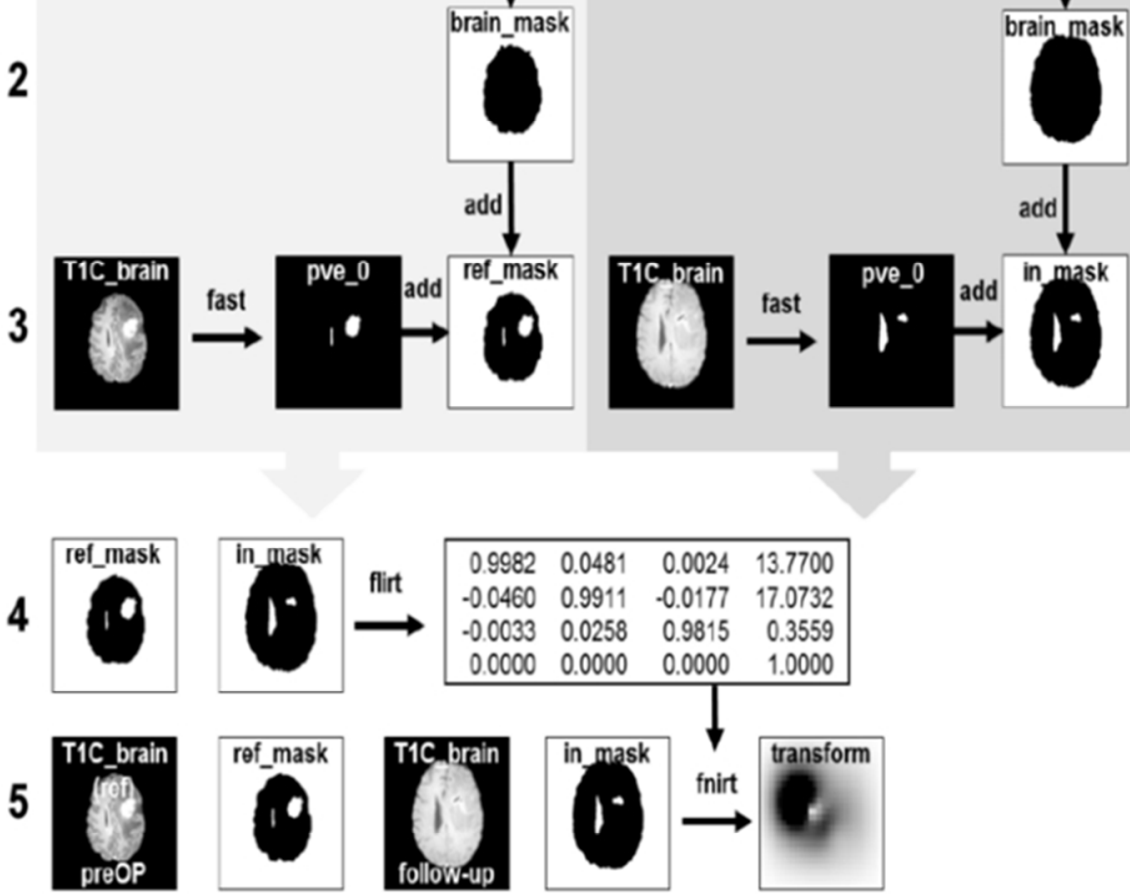

\begin{tabular}{|rrrr|}
\hline 0.9982 & 0.0481 & 0.0024 & 13.7700 \\
-0.0460 & 0.9911 & -0.0177 & 17.0732 \\
-0.0033 & 0.0258 & 0.9815 & 0.3559 \\
0.0000 & 0.0000 & 0.0000 & 1.0000 \\
\hline
\end{tabular}
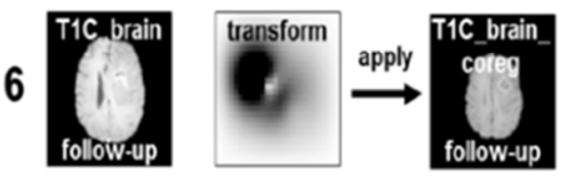

FIGURE 1 - Coregistration steps

The steps for the semi-automatic coregistration of the follow-up image with the preoperative reference image are illustrated. Number of the steps and filenames correspond to the text in the supporting material. The corresponding code can also be found in the text of the supporting material. 
Figure 2
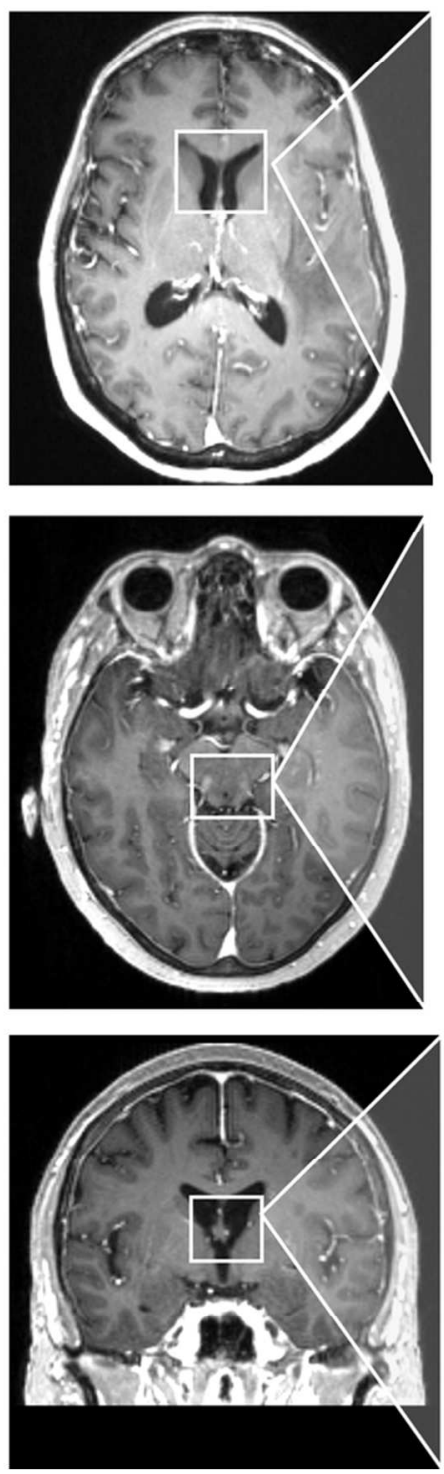

The top of the figure
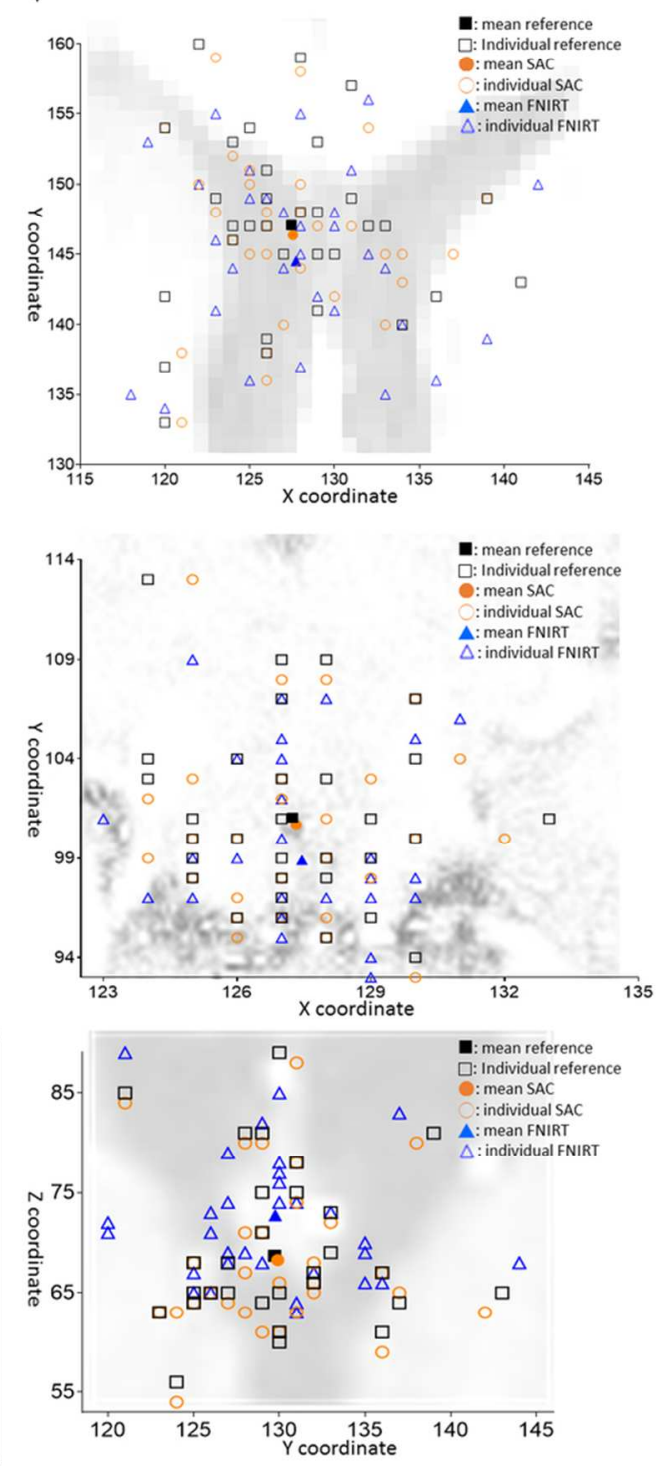

FIGURE 2 - Targeted registration errors

Targeted registration errors are shown for the septum pellucidum on axial view (A), cerebral aqueduct on axial view (B) and the uppermost of the third ventricle (C). Images on the left represent an individual case to show the location of targets and the graphs on the right correspond to the whole patients set.

Squares indicate coordinates for the presurgical MRI. The group mean is indicated by a closed square (') and individual patients by open squares $(\square)$. Circles indicate the coregistered image differences after semiautomatic nonlinear coregistration (SAC) of post- and preoperative images. The group mean is indicated by

a closed circle $(\bullet)$ and individual patients were indicated by open circles ( $\odot$ ). Triangles indicate the coregistered image difference after FNIRT nonlinear coregistration of post- and preoperative images. The group mean is indicated by a closed triangle $(\boldsymbol{\Lambda})$ and individual patients were indicated by open triangles $(\Delta)$.

$71 \times 98 \mathrm{~mm}(300 \times 300 \mathrm{DPI})$ 
Page 47 of 52

NMR in Biomedicine - For Peer Review Only

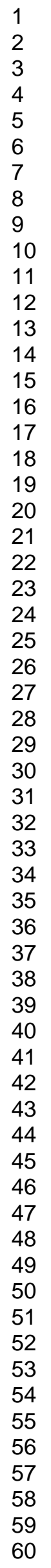




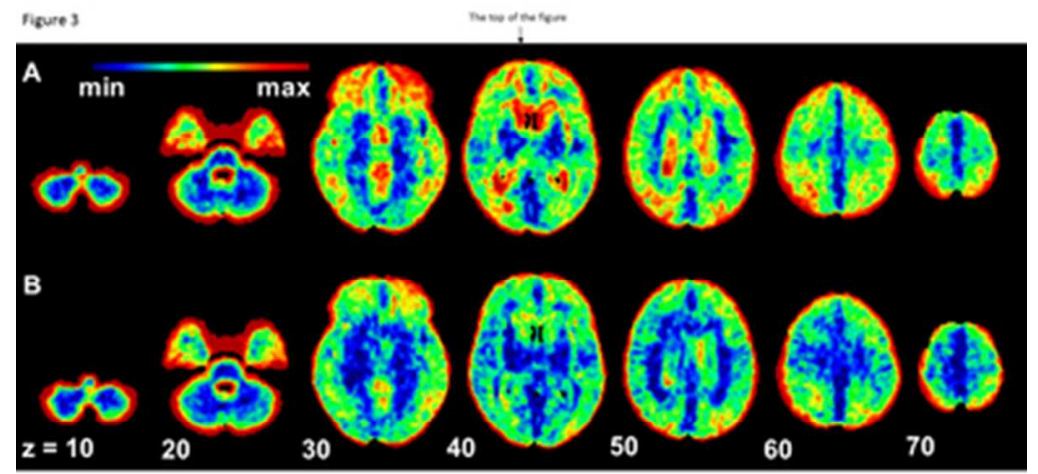

FIGURE 3 - Mean 3D group structural similarity for coregistration The group mean 3D structural similarity of all 32 cases is shown for the direct postoperative MRI (A) and later follow-up MRI at the time-point of tumor recurrence (B). Both coregistered to the preoperative MRI. Values indicate relative structural similarity between the coregistered and original preoperative scans for the group mean, with higher values indicating a greater similarity between the images being compared. Color bar showed the degree of similarity from totally identical (max, red) to $0 \%$ similar (min, black). $32 \times 18 \mathrm{~mm}$ (300 x 300 DPI) 

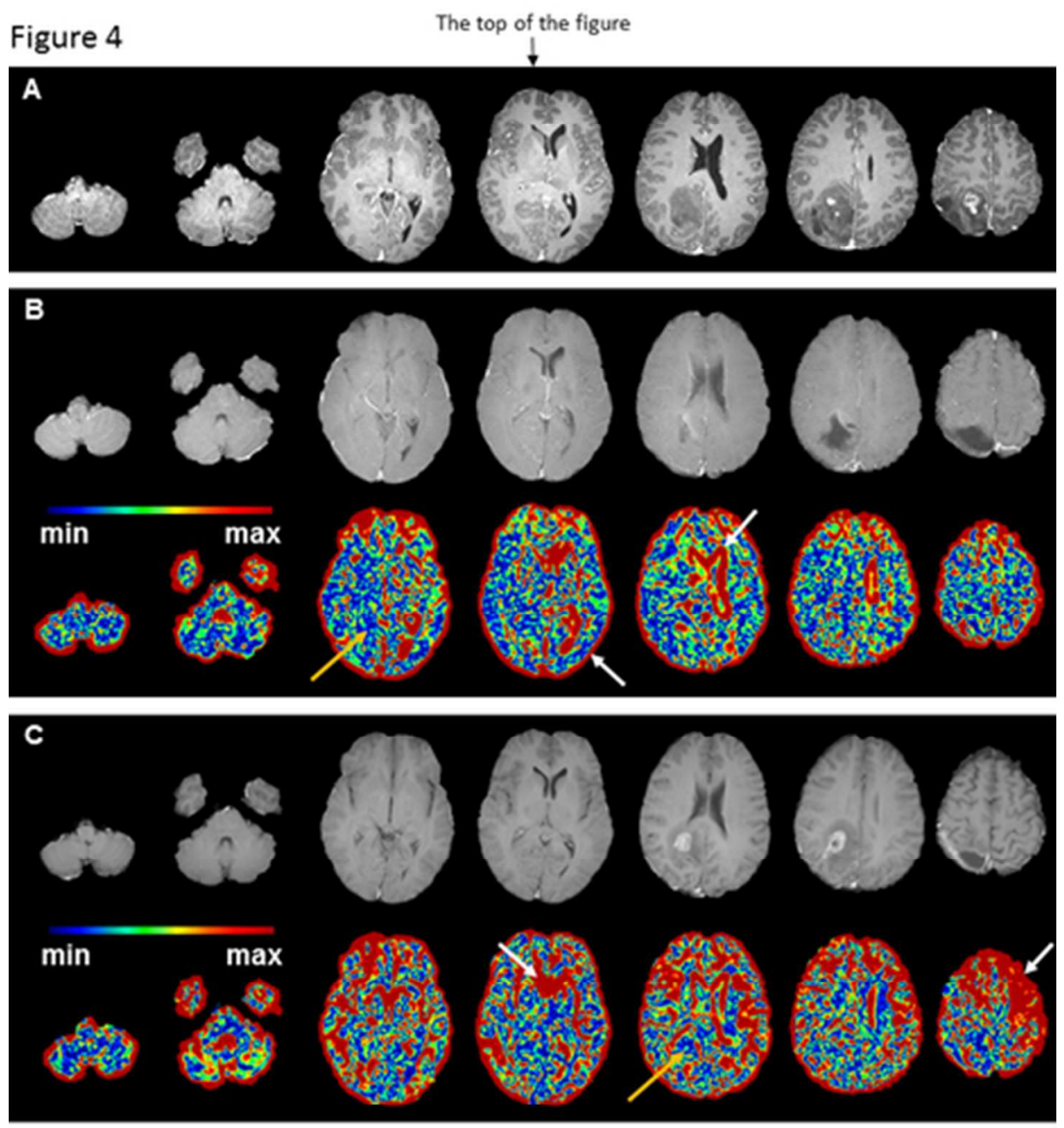

FIGURE 4 - Example of preoperative, direct postoperative and recurrent MRI coregistration An example of a representative patient with glioblastoma is shown. The preoperative reference scan $(A)$ is compared to coregistered postoperative scan (B) and recurrent scan (C), respectively. The color maps show the similarity between different time points, with red indicating higher and blue indicating a lower similarity index. Color bar showed the degree of similarity from totally identical (max, red) to $0 \%$ similar (min, black). These comparisons demonstrate a good performance of the semi-automatic coregistration method, especially in the peripheral area and ventricle area (white arrow), while lower performance is observed in the brain parenchyma and tumor area (brown arrow). $39 \times 50 \mathrm{~mm}(300 \times 300 \mathrm{DPI})$ 

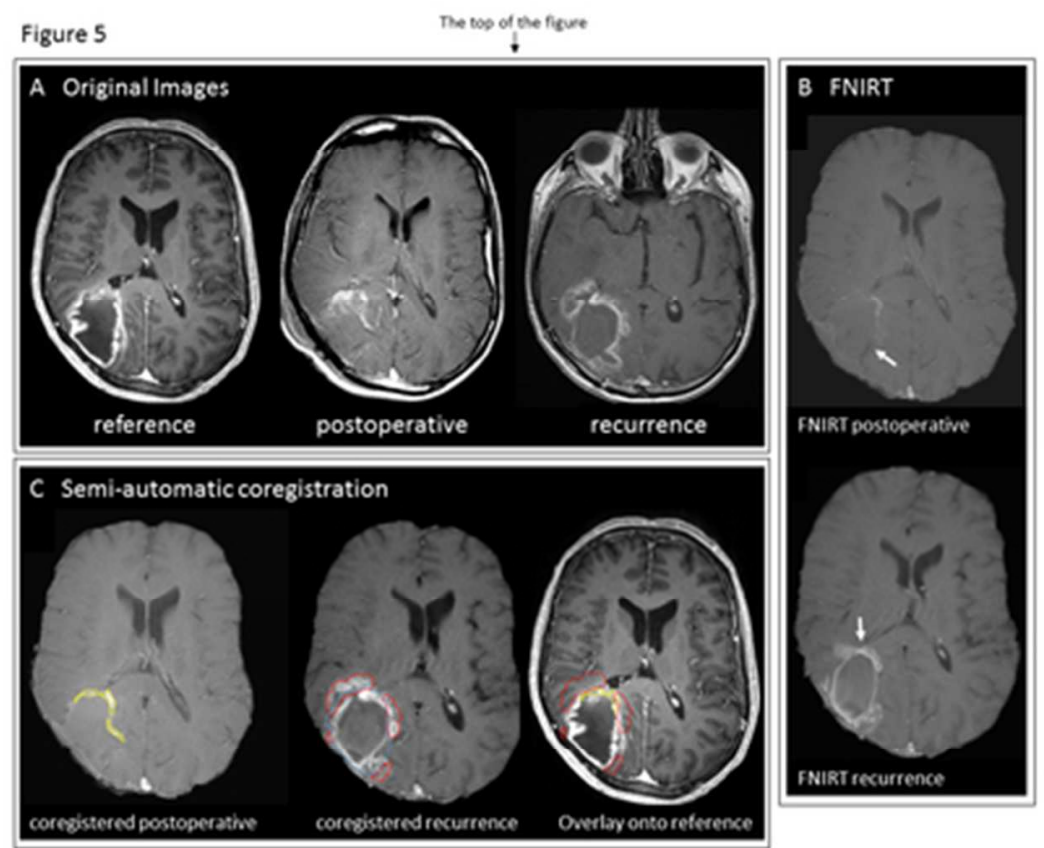

FIGURE 5 - Examples the of two-staged semi-automatic nonlinear coregistration Without coregistration, the postoperative and recurrent images are difficult to compare with the preoperative reference image (A). With standard FNIRT coregistration (B), although the gross brain positions are realigned in both postoperative and recurrent images, regional torsion, especially around the lesion (white arrow), may happen. After semi-automatic coregistration (C), postoperative residual regions (C, left, yellow outline) and recurrent regions $(C$, middle, red outline) can be fit with the preoperative reference image ( $C$, right). $34 \times 29 \mathrm{~mm}(300 \times 300$ DPI $)$ 


\section{SUPPORTING MATERIAL (for online-only publication)}

Steps and code of a semi-automatic method to coregister a preoperative MRI image of a patient with a brain tumor to follow-up images, for example direct or later postoperative. The steps are illustrated in Figure 1 with corresponding step numbers.

1. Brain extraction of the preoperative and follow-up T1-weighted scans with gadolinium Code: bet T1C T1C_brain -f 0.5 -B

Explanation: (bet $<$ in $><$ out $><$ options $>$ ). Input is an original T1-weighted image $\langle\mathrm{T} 1 \mathrm{C}\rangle$. A brain extracted image $<T 1 C$ _brain $>$ and binary brain mask <brain_mask $>$ will be created. The brain mask can be manually corrected using fslview.

2. The brain_mask is inverted creating the brain contour images Code: fslmaths brain_mask -mul -1 -add 1 T1C_brain_mask_inv Explanation: (fslmaths <input> <options> <output>). The image is multiplied by -1 and 1 is added.

3A. Segmentation to identify ventricles and enhancing tumor area or resection cavity for preoperative and follow-up scans

Code: fast -t 1 -n 4 -o T1C_brain T1C_brain

Explanation: (fast <options> <output><input>). Input is the previously created brain extracted T1-weighted image $<$ T1C_brain>. Segmentation creates a map of the ventricles and contrast enhancing tumor region $\langle$ pve_ 0$\rangle$. It is recommended to check this step and correct manually if needed.

3B. Create binary mask of brain contour, ventricles and lesion for preoperative and follow-up scans Code: fslmaths brain_mask_inv -add pve_0 (ref/in)_mask

Explanation: (fslmaths <in> <option> <in> <output>). The brain contour is added to the ventricles and lesion.

4. Create linear transformation matrix

Code: flirt -ref pre/ref_mask -in followup/in_mask -out FL_followupT1C_2_preT1C -omat FL_followupT1C_2_preT1C.mat -cost normmi -searchrx -90 90 -searchry -90 90 -searchrz -90 90 dof 12 -interp trilinear

Explanation: Reference image <-ref> is the preoperative binary mask of the brain contour, ventricles and contrast enhancing lesion. Input image <-in> is the follow-up binary mask of the 
brain contour, ventricles and resection cavity. Output <-omat> is the matrix used in the next step.

5. Create nonlinear transformation matrix which includes binary mask as input

Code: fnirt --ref=pre/T1C_brain --refmask=pre/ref_mask --in=followup/T1C_brain

--inmask=followup/in_mask --aff=FL_followupT1C_2_preT1C.mat $\quad$--cout=transform

--config=config.cnf

Explanation: The reference image <--ref> is the preoperative T1-weighted image. The reference mask <--refmask> is the binary mask of the brain contour, ventricles and contrast enhancing lesion. The input images are the follow-up T1-weighted scan <--in> and binary mask of the follow-up brain contour, ventricles and resection cavity <--inmask>. Output <--cout> is a transformation matrix.

6. Coregister brain from the follow-up scan with the preoperative scan

Code: applywarp --ref=pre/T1C_brain $\quad$--in=followup/T1C_brain $\quad$--warp=transform --out=T1C_brain_coreg

Explanation: The reference <--ref> is the preoperative brain extracted scan. Input <--in> is the brain extracted follow-up scan. The transformation matrix <--warp> was formed in the previous step. 
2

Graphic Abstract

A two stage semiautomatic non-linear coregistration method was proposed to coregistered brain MRI with high grade glioma before and after operation. Targeted registration error showed fair deviation both coregistered postoperative and follow-up recurrent images. A mean 3D structure

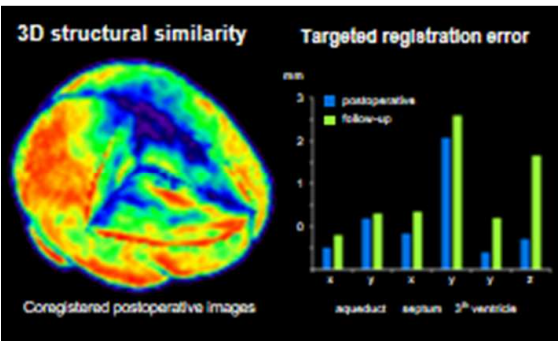

Validation of a semi-automatic coregistration of MRI scans in brain tumor patients during treatment follow-up

Anouk van der Hoorn, MD PhD ${ }^{1-3^{\dagger}}$; Jiun-Lin Yan, $\mathrm{MD}^{1,4-5^{+*}}$; Timothy J Larkin, $\mathrm{PhD}^{1}$; Natalie R Boonzaier, $\mathrm{MSc}^{1}$, Tomasz Matys, similarity also demonstrate optimal result. 